\title{
A numerical simulation of metallic cylindrical sandwich shells subjected to air blast loading
}

\begin{abstract}
The dynamic response of cylindrical sandwich shells with aluminum foam cores subjected to air blast loading was investigated numerically in this paper. According to KNR theory, the nonlinear compressibility of the air and finite shock conditions were taken into account in the finite element model. Numerical simulation results show that the compression strain, which plays a key role on energy absorption, increases approximately linearly with normalized impulse, and reduces with increasing relative density or the ratio of face-sheet thickness and core thickness. An increase of the impulse will delay the equalization of top and bottom face-sheet velocities of sandwich shell, but there is a maximum value in the studied bound. A limited study of weight optimization was carried out for sandwich shells with respect to the respective geometric parameters, including face-sheet thickness, core thickness and core relative density. These numerical results are of worth to theoretical prediction and engineering application of cellular metal sandwich structures.
\end{abstract}

\section{Keywords}

Dynamic response, Sandwich shell, Blast loading, Numerical simulation, Optimal design
Lin Jing ${ }^{a, b}$,

Fei Yang ${ }^{c}$,

Zhihua Wang ,

Longmao Zhao ${ }^{\mathrm{a}, *}$

Institute of Applied Mechanics and Biomedical Engineering, Taiyuan University of Technology,

Taiyuan 030024, China

${ }^{b}$ Impact Mechanics Laboratory, Department of Mechanical Engineering, National University of Singapore, Singapore 117576, Singapore

College of Science and Engineering, Jinan

University, Guangzhou 510632, China

Received 01 Aug 2012

In revised form 19 Dec 2012

Author email: zhaolm@tyut.edu.cn (LM Zhao) jinglin_426@163.com (L Jing)

\section{INTRODUCTION}

Cellular metals including foams and honeycombs, as a new class of ultra-light multi-functional materials, are widely used as advanced structural components in many engineering applications due to their excellent physical, thermal and mechanical properties [1-3]. Extensive applications of cellular metals include light weight cores for sandwich structures to increase the shock resistance, and improve the energy absorbing capacity. The sandwich structure is a special topology form comprising a combination of different materials that are bonded to each other so as to utilize the properties of each component for the structural advantage of the whole assembly. Potential advantages of sandwich beams or panels with two metallic face-sheets and a cellular metal core over solid structures of equal mass under intensive impact/blast loadings have been massively studied in the literatures [410]. Typical deformation and failure modes, such as face-sheet yield, core compression and shear, 
have been observed in experimental tests, while prediction of load-bearing capacity, failure mechanisms and energy dissipation, have been examined theoretically and numerically. Sandwich shell structure, a better combination of the advantages of the solid monolithic shell and sandwich structure, is expected and would thereby be of great importance for its applications. However, studies on curved sandwich structures appear quite limited to date. Shen et al [11] undertook an experimental investigation into curved sandwich panels with aluminum foam cores subjected to air blast, and demonstrated that the performance of sandwich shells is superior to that of solid counterparts of equivalent mass. Using finite element simulations, Liu et al [12] investigated the dynamic response and blast resistance of metallic sandwich-walled hollow cylinders with graded aluminum foam cores. Recently, Hoo-Fatt et al [13] developed an analytical solution for the early time response of a composite sandwich cylindrical shell subjected to external blast. Frostig et al [14] developed a non-linear analysis of a delaminated curved sandwich panel with a compliant core, subjected to a thermal field and mechanical loading or combined. It can be concluded from these existing literatures that there is yet no comprehensive report on the energy absorption and optimal design of metallic cylindrical sandwich shells under air blast loading.

Usually, the dynamic response of the sandwich structures subjected to blast/shock loading is usually split into three sequential steps [15]: stage I is the one-dimensional fluid-structure interaction problem, and results in a uniform velocity of the front face sheet; during stage II the core compresses and stage III is structure bending and stretching phase. Although fluid-structure interaction has been shown to significantly enhance the relative performance of sandwich structures, it has little previously been taken into account in assessing relative structure performance [8]. For one thing, the effect is small on the base of the Taylor's linear theory [4], for another, the theoretical results for intense air blasts is few. As in Taylor's model, the fluid is assumed to undergo relatively small volume changes and the plate is idealized as an unsupported planar surface with mass/area, and the resistance from the air behind the plate is neglected. Earlier experimental study [9] showed that the momentum transmitted to the front face sheet with pressure as large as several tens $M P a$, but still below levels at which non-ideal gas effects begin to become important. Therefore, the nonlinear compressibility of the air and finite shock conditions must be taken into account in assessing the response of sandwich structures subjected to intense air shocks. Kambouchev et al [16] have extended the Taylor's theory of fluid-structure interaction for air blasts by accounting for nonlinear compressibility and finite shock behavior. The results were employed by Vaziri [8] to investigate the dynamic performance of metal sandwich plates in air blasts. Based on these researches, the nonlinear compressibility of the air and finite shock conditions were taken into account in the FE model, to investigate the dynamic response, energy absorption and optimal design of metallic cylindrical sandwich shells subjected to intense shock loading in this paper.

\section{FLUID-STRUCTURE INTERACTION IN KNR THEORY}

Kambouchev et al [16] have extended the Taylor's linear theory for air blasts, which is referred to as KNR theory in this paper. KNR theory accounts for nonlinear compressibility of the air and finite shock conditions. The one-dimensional interaction between a linear wave of form (1), which

Latin American Journal of Solids and Structures 10(2013) $631-645$ 
decays approximately exponentially in time with time constant, $t_{0}$, with a plate of density $\varrho_{p}$ and thickness $h_{p}$.

$$
p=p_{0} e^{-t / t_{0}}
$$

where, $p_{0}$ is the maximum overpressure of the blast wave and $t_{0}$ is the decay period.

The peak density, $\varrho_{0}$, just behind the shock in the incident wave is

$$
\frac{\rho_{0}}{\rho_{A}}=\frac{7+6\left(p_{0} / p_{A}\right)}{7+\left(p_{0} / p_{A}\right)}
$$

and the speed of the shock front, $c_{s}$, is

$$
\frac{c_{s}}{c_{A}}=\sqrt{\frac{6 p_{0}}{p_{A}}+1}
$$

where, $p_{A}, c_{A}, \varrho_{A}$ are the ambient atmospheric pressure, sound speed and density, respectively.

The common method for studying air blast loads on a structure is to assume that the structure is rigid and fixed, and the momentum per unit area of the incident wave is

$$
I=\int_{0}^{\infty} p(t) d t=p_{0} t_{0}
$$

But it is not in the nonlinear KNR theory. It is assumed that a constant atmospheric pressure $p_{A}$ is applied on the back side of the plate to ensure that the plate is initially in equilibrium. Upon integration of the equation of motion of the plate subject to the requirement of continuity with the motion of the fluid, the resulting pressure acting on the plate $p_{p}$ is obtained as

$$
p_{p}=\frac{2 p_{0}}{\beta_{0}-1}\left[\beta_{0} \exp \left(-\beta_{0} \frac{t}{t_{0}}\right)-\exp \left(-\frac{t}{t_{0}}\right)\right]
$$

where

$$
\beta_{0}=\frac{t_{0}}{t_{s}^{*}}
$$

with

$$
t_{s}^{*}=\frac{\rho_{p} h_{p}}{\rho_{0} c_{s}}
$$


The pressure continuously decreases as the plate accelerates and vanishes at time

$$
t_{v}=t_{0} \frac{\log \beta_{0}}{\beta_{0}-1}
$$

At this point, the plate velocity reaches a maximum and so does the transferred momentum per unit area of the plate $I_{p}$, which adopts a value

$$
\frac{I_{p}}{I}=2 \beta_{0}^{\beta_{0} /\left(1-\beta_{0}\right)}
$$

In the simulations presented in this paper, the applied pressure approach, applies the following time-dependent pressure history to the plate surface towards the blast, is employed for modeling a uniform planar wave striking a plate

$$
p=C_{R} p_{0} e^{-t / t_{R}} \text { with } \frac{t_{R}}{t_{0}}=\frac{I}{C_{R} p_{0} t_{0}}
$$

where,

$$
C_{R}=2 \frac{7+4\left(p_{0} / p_{A}\right)}{7+\left(p_{0} / p_{A}\right)}
$$

For small $p_{0} / p_{\mathrm{A}}, C_{R} \cong 2$, while, for $p_{0} / p_{\mathrm{A}}>>1, C_{R} \rightarrow 8$.

In this paper, the additional parameter choices are as follows: $t_{0}=10^{-4} \mathrm{~s}, p_{A}=0.1 \mathrm{MPa}, \varrho_{A}=1.25 \mathrm{Kg} / \mathrm{m}^{3}, c_{A}=331 \mathrm{~m} / \mathrm{s}, p_{0}=10 \mathrm{MPa}$, and $C_{R}=8$.

\section{FINITE ELEMENT MODEL}

In this section, the numerical simulation that employs the above applied pressure approach for modeling the air blast loading to the peripherally clamped sandwich shells was conducted on the base of KNR theory. Consider a sandwich shell (310 $\mathrm{mm}$ long, with an arc length also of $310 \mathrm{~mm}$ ) of span $2 L=250 \mathrm{~mm}$, radius $R=250 \mathrm{~mm}$ with face-sheet thickness $h_{f}$ and core thickness $h_{c}$. The numerical model was made using the non-linear, explicit FE code LS-DYNA (version 970). Since the sandwich shell is symmetric about $x-z$ and $y$ - $z$ planes, only a quarter of the shell was modeled, as shown in Fig.1. To balance the computational accuracy and efficiency, the central area of sandwich shells was meshed into $1 \mathrm{~mm} \times 1 \mathrm{~mm}$ while the other area was meshed into $2 \mathrm{~mm} \times 2 \mathrm{~mm}$. The transition parts of the two areas with different mesh sizes were connected artificially using the triangular elements. The entire model comprises 53166 nodes and 61257 elements. The LY-12 face-sheets were modeled by Belytschko-Tasy shell element. The foam core was modeled by the default brick element. Hourglass control was used for both shells and bricks.

Latin American Journal of Solids and Structures 10(2013) $631-645$ 
The mechanical behavior of LY-12 aluminum alloy face-sheets were represented by material model 3 of LS-DYNA (*MAT_PLASTIC_KINEMATIC). Due to the rate-insensitive of aluminum alloy, strain rate effects are ignored in the simulation. The aluminum foam was modeled by material model 63 of LS-DYNA (*MAT_CRUSHALBE_FOAM), which is dedicated to modeling crushable foam with optional damping and tension cutoff. In this model, tension is treated as elasticperfectly-plastic at the tension cut-off value.

The specimens were clamped along their periphery by steel frames and bolts in the tests while that was represented by nodal constraints in the numerical model, leaving a curved exposed area of $250 \mathrm{~mm} \times 250 \mathrm{~mm}$. A given pressure pulse loading was applied onto the top face-sheet of sandwich shells based on the KNR theory, and the loading parameters were presented in Section 2. Automatic, surface-to-surface contact options were generally used. It should be pointed out that the analyses of the effect of element size on the structural response of sandwich shells were omitted in this study since the similar/same methods was employed in some previous studies and a good agreement was obtained comparing with that of experimental results $[7,17,18]$.

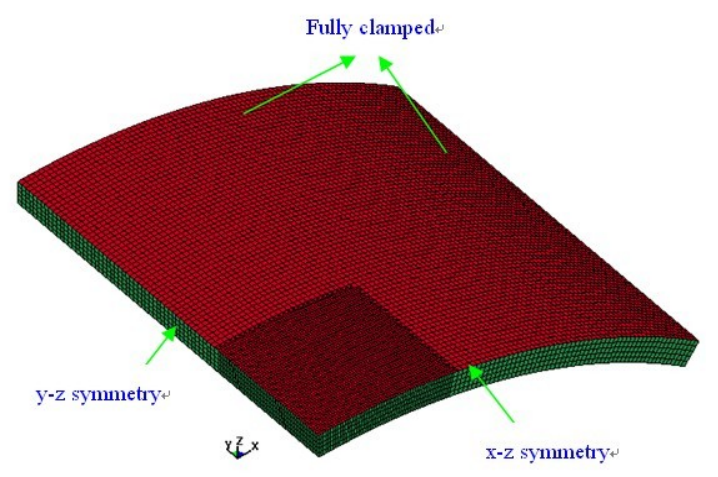

Figure 1 FE model of $1 / 4$ sandwich shell

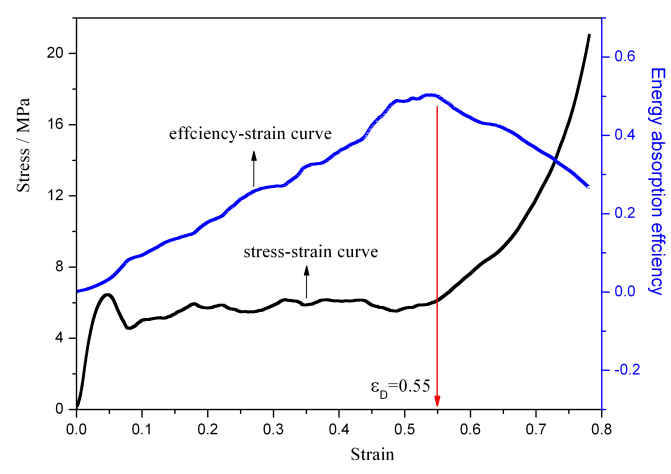

Figure 2 Stress-strain curve of foam core

Table 1 LS-DYNA material type, material property input data

\begin{tabular}{|c|c|c|}
\hline Material & Part name & $\begin{array}{l}\text { LS-DYNA material type and material property } \\
\text { input data (unit }=\mathrm{cm}, \mathrm{g}, \mu \mathrm{s}, \mathrm{Mbar})\end{array}$ \\
\hline \multirow{3}{*}{$\begin{array}{l}\mathrm{LY}-12 \\
\text { aluminum alloy }\end{array}$} & Face-sheet & ${ }^{*} \mathrm{MAT}_{-} \mathrm{PLASTIC} \_$KINEMATIC \\
\hline & & ETAN \\
\hline & & $0.68 \quad 0.33 \quad 3.1 \mathrm{E}-3 \quad 4.62 \mathrm{E}-2$ \\
\hline \multirow[t]{4}{*}{ Aluminum foam } & Core & *MAT_CRUSHABLE_FOAM \\
\hline & & LCID \\
\hline & & DAMP \\
\hline & & $\begin{array}{lll}0.485 & 0.02 & 0.0\end{array}$ \\
\hline
\end{tabular}

The standard quasi-static tests were conducted for LY-12 aluminum alloy face-sheet and aluminum foam core material in the corresponding experimental investigations [19, 20] to obtain the cor- 
responding mechanical parameters. The uniaxial compressive stress versus strain curve of closed-cell aluminum foam with relative density of $18 \%$ is plotted in Fig.2, while the material properties of aluminum alloy face-sheet were listed in Table 1. Here, an energy efficiency-based approach is proposed to calculate the plateau stress and densification strain of foam core. Energy absorption efficiency $\eta\left(\varepsilon_{a}\right)$ is defined as the energy absorbed up to a given nominal strain $\varepsilon_{a}$ normalized by the corresponding stress value $\sigma_{c}(\varepsilon)[21]$ :

$$
\eta\left(\varepsilon_{\alpha}\right)=\frac{\int_{\varepsilon_{\beta}}^{\varepsilon_{\alpha}} \sigma_{c}(\varepsilon) d \varepsilon}{\sigma_{c}(\varepsilon)_{\varepsilon=\varepsilon_{\alpha}}}
$$

where $\varepsilon_{c r}$ is the strain at the initial yield point corresponding to the start of the plateau regime. Densification strain $\varepsilon_{D}$ is the strain value corresponding to the stationary point in the efficiencystrain curve where the efficiency is a global maximum, i.e.

$$
\left.\frac{d \eta(\varepsilon)}{d \varepsilon}\right|_{\varepsilon=\varepsilon_{D}}=0
$$

The energy absorption efficiency curve of aluminum foam is also shown in Fig. 2. The plateau stress is obtained by

$$
\sigma_{p l}=\frac{\int_{\varepsilon_{c r}}^{\varepsilon_{a}} \sigma_{c}(\varepsilon) d \varepsilon}{\varepsilon_{a}-\varepsilon_{c r}}
$$

For the tested aluminum foam specimen, its plateau stress $\sigma_{p l}$ and densification strain $\varepsilon_{D}$ are 5.49 $M P a$ and 0.55 , respectively.

It is noted that, generally, the objective of optimal design of sandwich structures with cellular metal cores is to obtain the maximum loading-carrying capability (i.e. minimize the permanent central point deflections of back face-sheet) by varying their geometrical configurations. However, for some specific situations such as the components used in aircrafts, these sandwich structures are desired to achieve the best shock resistance performance for a given mass. Thus, this study presents a near-optimal design for a sandwich shell structure with a given mass.

The normalized mass per unit area of the shell, $\bar{M} / \rho_{f} L$, is given by

$$
\frac{\bar{M}}{\rho_{f} L}=2 \frac{h_{f}}{L}+\bar{\rho} \frac{h_{c}}{L}
$$

To eliminate the effect of mass on the structural response, all numerical simulations conducted in this paper are performed for sandwich shells with $\bar{M} / \rho_{f} L=0.025$ and $L=125 \mathrm{~mm}$. 


\section{RESULTS AND DISCUSSION}

\subsection{Deformation process}

Fig. 3 shows a typical deformation process of metallic cylindrical sandwich shells with aluminum foam cores subjected to intensive shock loading. When the momentum was transmitted to the top face-sheet of sandwich shell structure, the top face-sheet obtained immediately an initial velocity, and then the core was compacted gradually by top face-sheet resulting in core compression deformation. Finally, the top face-sheet, core and bottom face-sheet achieved a common velocity while a large amount of energy was dissipated by the large deformation of top face-sheet and core compression. The sandwich shell continued to deform under its own inertia and the maximum deformation deflection took place at the time $t=420 \mu \mathrm{s}$. Henceforth, a slight oscillation of the shell occurred with the deformation, and the structure was finally brought to rest by plastic bending and stretching. The final shape of deformation profile of sandwich shell structures is a proximate dome.
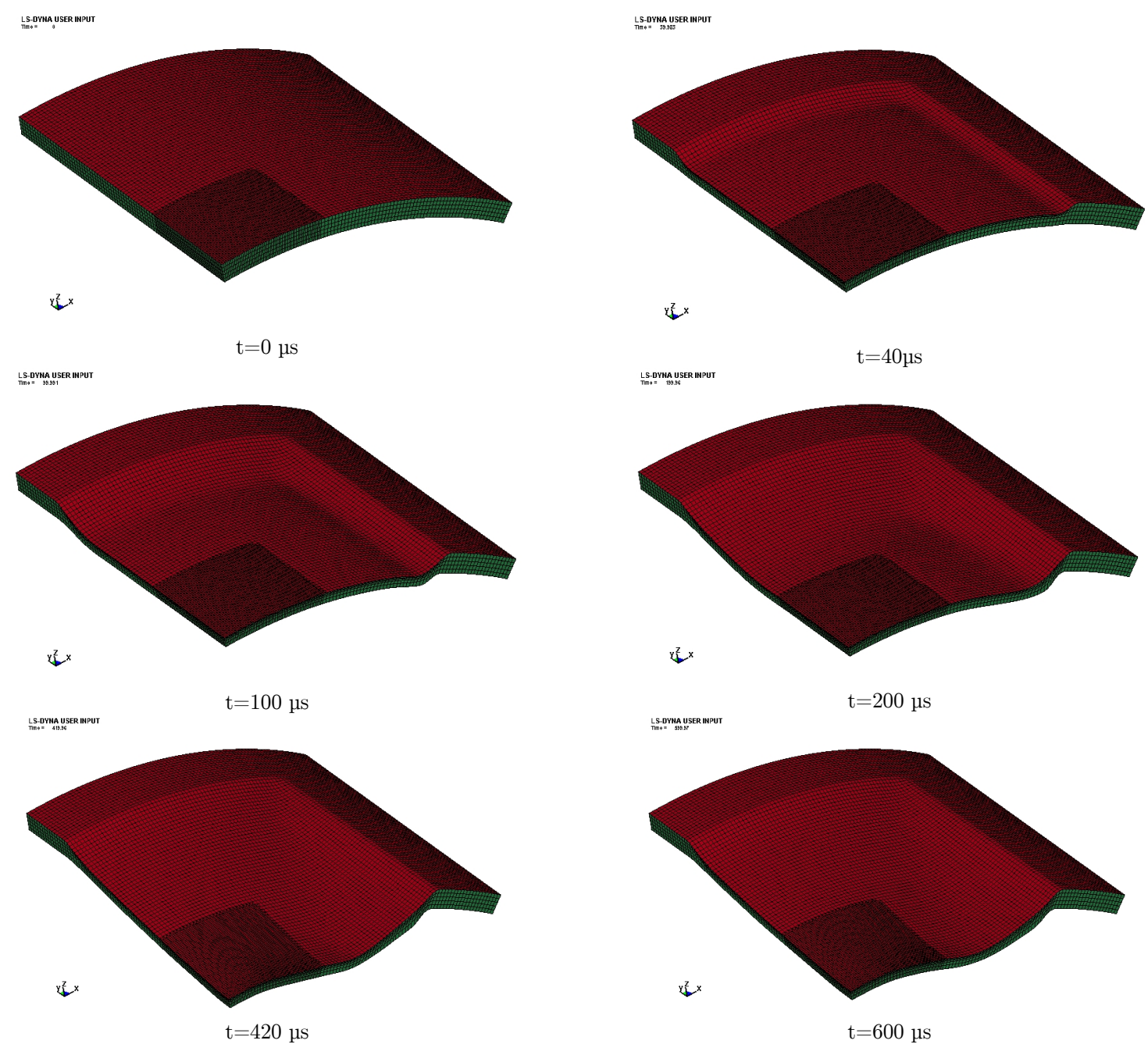

Figure 3 A typical deformation process of metallic sandwich shell structures $\left(\bar{I} /\left(\bar{M} \sqrt{\sigma_{f Y} / \rho_{f}}\right) \approx 0.0845\right)$ 


\subsection{Dynamic response}

Sandwich structures with cellular metal cores such as honeycombs or aluminum foams have the capability of dissipating considerable energy by large core compression deformation under impact/blast loading. Therefore, core compression strain $\varepsilon_{c}$ is a key parameter for evaluating the energy absorption capability of sandwich structures. The core compression strain $\varepsilon_{c}$ of sandwich shells with aluminum foam cores was plotted in Fig. 4 as a function of the normalized impulse $\bar{I} /\left(\bar{M} \sqrt{\sigma_{f Y} / \rho_{f}}\right)$ and the structural configurations $\left(h_{f} / h_{c}\right.$ and $\left.\bar{\rho}\right)$. It can be found that the compression strain $\varepsilon_{c}$ increases approximately linearly with normalized impulse, and reduces with increasing relative density $\bar{\rho}$ and the ratio of face thickness and core thickness $h_{f} / h_{c}$. It is noted that the core compression strain $\varepsilon_{c}$ is not sensitive to $h_{f} / h_{c}$ in the studied bound.

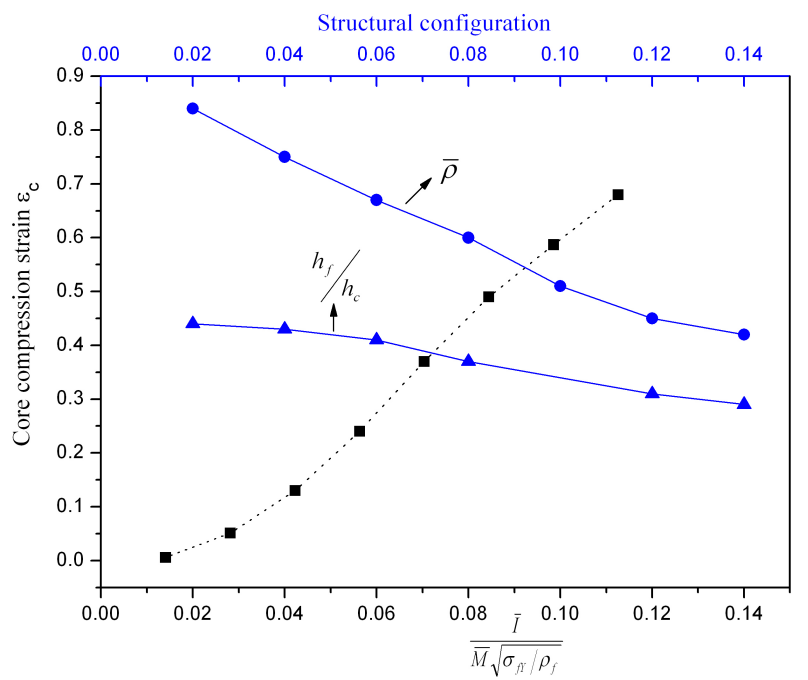

Figure 4 The core compression strain as a function of the normalized impulse and the structural configurations

One of the key points to resist impact objects is to efficiently dissipate the kinetic energy of the blast loading. Based on the analytical mode, in the stage I, the impulse delivered onto the sandwich shell $(I)$ is assumed to have a uniform distribution over the top face. The corresponding kinetic energy of the top face is obtained by

$$
W_{1}=\frac{I^{2}}{2 A_{0} \rho_{f} h_{f}}
$$

where $A_{0}$ is exposed area of the shell. $\varrho_{f}$ and $h_{f}$ are material density and thickness of the face-sheets, respectively. At the end of this stage, the top and bottom face-sheets as well as the core structure all have identical velocity

$$
v_{2}=\frac{I}{A_{0}\left(2 \rho_{f} h_{f}+\rho_{c} h_{c}\right)}
$$


where $\varrho_{c}$ is mass density of core material, and $h_{c}$ is core thickness.

Correspondingly, the kinetic energy of the entire structure at the end of stage II is written as

$$
W_{2}=\frac{I^{2}}{2 A_{0}\left(2 \rho_{f} h_{f}+\rho_{c} h_{c}\right)}
$$

Hence, the energy absorption in core compression is

$$
E_{p}=W_{1}-W_{2}
$$

or

$$
\frac{W_{1}-W_{2}}{W_{1}}=\frac{1+\mu}{2+\mu}
$$

with $\mu=\left(\rho_{c} h_{c}\right) /\left(\rho_{f} h_{f}\right)$ being the ratio of core mass and face mass.

Using the dynamic plateau stresses, the energy dissipation during core crushing also can be obtained by

$$
E_{p}=A_{0} \sigma_{p l}^{d} \Delta C
$$

where $\sigma_{p l}^{d}$ is the dynamic plateau stress of foam core, and $\sigma_{p l}^{d}=\phi \sigma_{p l}, \phi$ is the ratio of dynamic and quasi-static plateau stress, $\sigma_{p l}$ is the quasi-static stress, and can be obtained from Eq. (14). $\Delta C$ is the amount of core compression. The aluminum foam used in the experiment did not exhibit evident strain rate effect and hence the dynamic plateau stress is taken the same as the quasi-static one.

To clarity the energy dissipation mechanism of cylindrical sandwich shells, Fig. 5 illustrates clearly the partition of energy absorption by cores and face sheets of sandwich shells subjected to different impulse levels. It is evident that the energy absorption by every component of specimens increases with normalized impulse, and the core compression plays the key role on energy absorption, which contributes over $2 / 3$ of the total dissipation. Increasing normalized impulse levels by $49.9 \%, 33.37 \%, 24.96 \%, 19.99 \%, 16.67 \%$ and $14.29 \%$ lead to a rise of total plastic energy dissipation in the shells. The increases in internal energy in every specimen are 83.11\%, 62.9\%, 48.36\%, $38.57 \%, 30.86 \%$ and $27.17 \%$, respectively, which are the approximate square roots of increases of the impulses. 


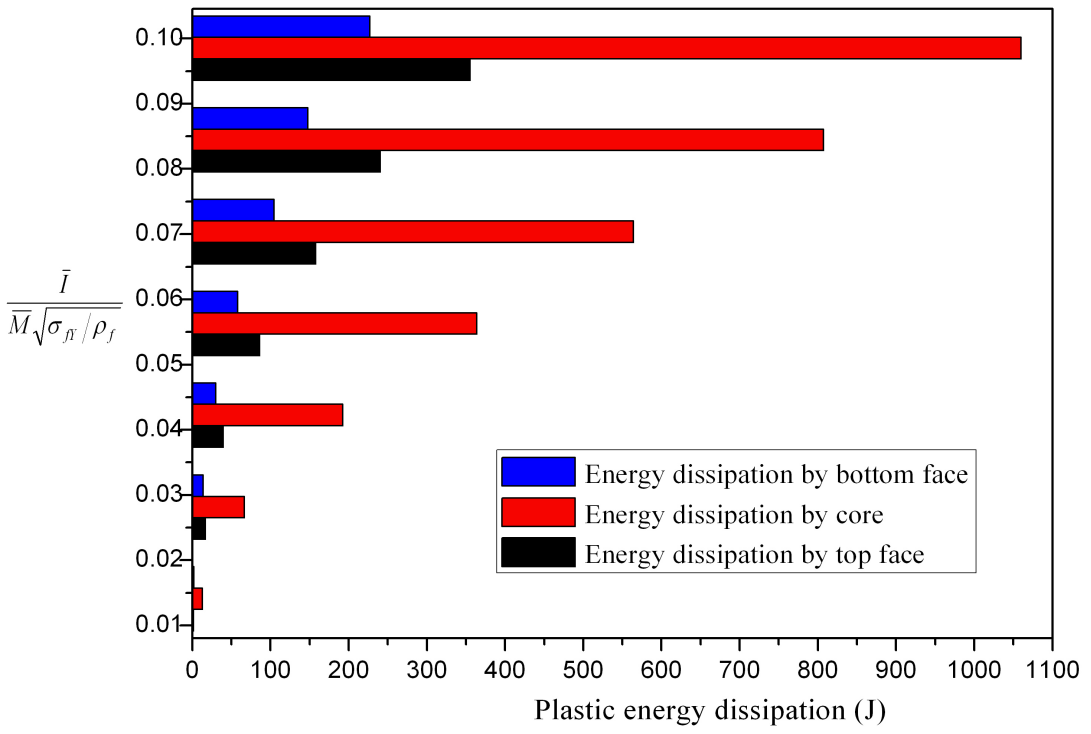

Figure 5 Plastic energy dissipation by face sheets and core of sandwich shells subjected to different impulse levels $\left(h_{c}=10 \mathrm{~mm}, h_{f}=0.8 \mathrm{~mm}\right.$ and $\bar{\rho}=0.18)$

Some FE simulations $[22,23]$ investigated the coupling effects between the stages of the response based on Fleck and Deshpande's model. The results suggest that this model may obtain the overestimate or under-estimate predicted deflection due to the fully decoupled assumption. Fig. 6 shows the velocity-time histories of the mid-span of the top and bottom face-sheets of sandwich shells subjected to different impulse levels. In the early stage of structural response, the velocity of top face-sheet reaches its maximum value and decreases gradually due to core compression until the top and bottom face-sheet velocities equalization. Subsequently, sandwich shells continue to move at a decreasing speed. In addition, blast loadings with different pressure magnitudes cause different relative motion of the top and bottom face-sheets of the sandwich shells, which lead to different core compression. Small loading impulse makes the velocities of top and bottom sheets equal rather early in the deformation history (as shown in Fig. 6a) when a small partial densification of the core has developed. An increase of the impulse (as shown in Fig. 6b) delays the equalization of top and bottom face velocities, which results in the possibility of forming a shock front in the core. It should be noted that there is a maximum $t_{e q}=0.068 m s$ for the normalized impulse $\bar{I} /\left(\bar{M} \sqrt{\sigma_{f y} / \rho_{f}}\right) \approx 0.1$, and exceed the loading impulse this time starts to decrease although the impulse increases further in the studied bound. It is interesting to note that the variations of the velocities of top and bottom facesheets after the time $t>t_{e q}$ are related to the applied loading. 


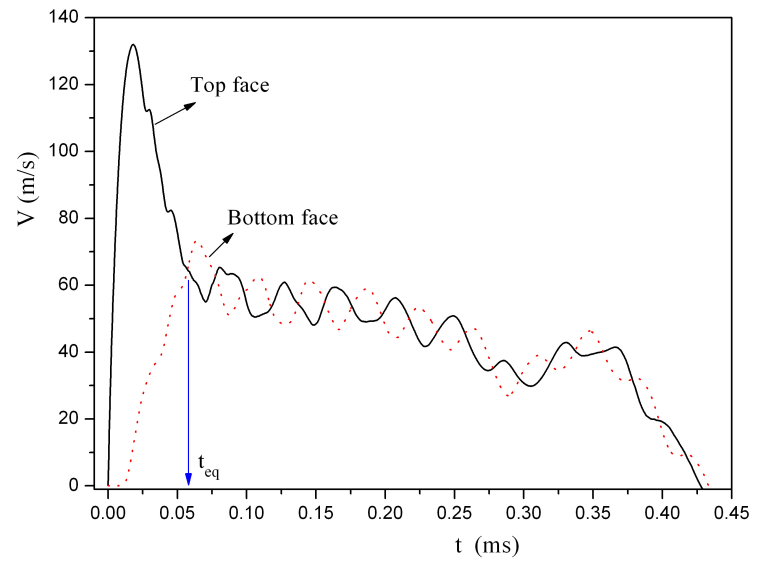

(a) $\bar{I} /\left(\bar{M} \sqrt{\sigma_{f Y} / \rho_{f}}\right) \approx 0.056$

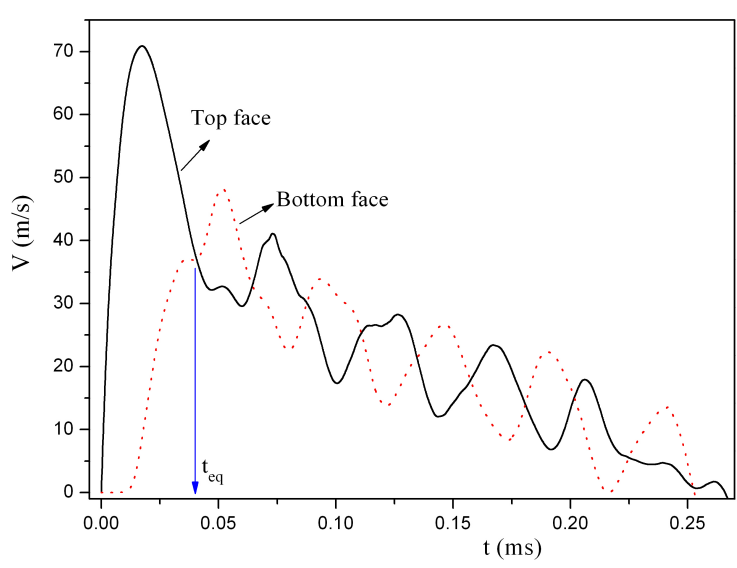

(b) $\bar{I} /\left(\bar{M} \sqrt{\sigma_{f Y} / \rho_{f}}\right) \approx 0.07$

Figure 6 velocity-time histories at the mid-span of the top and bottom face of sandwich shells $\left(h_{c}=10 \mathrm{~mm}, h_{f}=0.8 \mathrm{~mm}\right.$ and $\left.\bar{\rho}=0.18\right)$

\subsection{Near-optimal design}

Preliminary studies $[5,7,9,11]$ show that the center-point deflection of sandwich structures is reduced by increasing the face-sheet thickness, core thickness and relative density and this however leads to an increase in the structural weight. How to make a compromise between strength and weight is one of the most important issues that need to be considered in the design of sandwich structures. So an effort to obtain near-optimal configurations for sandwich shells under blast load was made, and all the results were presented in a dimensionless form. It should be noted that when the mass and span of the sandwich shell are specified, the geometry of the sandwich shell is specified by three variables, i.e. face-sheet thickness, core thickness and core relative density. The facesheet thickness is then determined in terms of core relative density and core thickness by Equation (15).

Similar to sandwich panels [24], we assume the normalized maximum deflection defends on the dimensionless variables according to

$$
\frac{W_{\max }}{L}=f\left[\frac{\bar{I}}{\bar{M} \sqrt{\sigma_{f Y} / \rho_{f}}}, \frac{\bar{M}}{\rho_{f} L}, \frac{h_{f}}{h_{c}}, \frac{h_{c}}{L}, \bar{\rho}, \alpha\right]
$$

Here, $a$ indicates a dependence on the radius of curvature of sandwich shells. For simplicity, the effect of curvature was neglected in this paper. 
Figs. 7 (a)-(c) present the normalized maximum deflection $W_{\max } / L$ of the top and bottom facesheets of sandwich shells for the fixed value of normalized mass $\bar{M} / \rho_{f} L=0.025$ as a function of the relative density $\bar{\rho}$, normalized core thickness $h_{c} / L$ and normalized impulse $\bar{I} /\left(\bar{M} \sqrt{\sigma_{f Y} / \rho_{f}}\right)$, respectively. In Fig. 7 (a), the relative density $\bar{\rho}$ of sandwich shells is varied for a given values of normalized core height and impulse $\left(h_{c} / L=0.08, \bar{I} /\left(\bar{M} \sqrt{\sigma_{f Y} / \rho_{f}}\right) \approx 0.07\right)$. As the relative density $\bar{\rho}$ increases from 0.02 to 0.20 , both the top and bottom face sheets experience the smallest maximum normalized deflection at a relative density $\bar{\rho}=0.06$. Typically, the normal compressive strain in the core, defined as compressive deformation per unit core thickness, is an important parameter to influence the shock resistance of sandwich structures by dissipating a large amount of energy. The foam cores near the center of sandwich shell have fully densified at this impulse level, but the foam cores near the edge of sandwich shell have not densified because of constraint of the clamped edges.

In Fig. 7 (b), the relative density is fixed at $\bar{\rho}=0.06$, the normalized impulse is fixed at $\bar{I} /\left(\bar{M} \sqrt{\sigma_{f Y} / \rho_{f}}\right) \approx 0.07$, and the normalized core thickness $h_{c} / L$ is varied. In this case, the thickness of face-sheets $h_{f}$ obtained from Eq. (15) increases with decreasing the core thickness $h_{c}$. When $h_{f} / h_{c}$ is much small, the structural performance of sandwich shells tends to that of monolithic aluminum foam shells due to extremely weak protection of face sheets. As $h_{f} / h_{c}$ increases, sandwich shell undergoes more and more global bending and core crushing wasn't obvious. From Fig. 7 (b), the core thickness significantly affects the maximum deflection of both top and bottom face-sheets, and the minimum value of center-point deflection is obtained for a relatively thin core with $h_{c} / L=0.06$ for $\bar{\rho}=0.06$ and $\bar{I} /\left(\bar{M} \sqrt{\sigma_{f Y} / \rho_{f}}\right) \approx 0.07$. Obviously, these results are sufficient to reveal that for such sandwich shells with $\bar{M} / \rho_{f} L=0.025$ and $R=250 \mathrm{~mm}$, the optimal structural configuration is $\bar{\rho}=0.06$ and $h_{c} / L=0.06$.

The numerical computations of maximum deflection for the sandwich shell having normalized mass $\bar{M} / \rho_{f} L=0.025$ were carried out at different impulse levels and presented in Fig. 7 (c). It is seen that the normalized center-point deflection of sandwich shells increases monotonically with normalized impulse. 


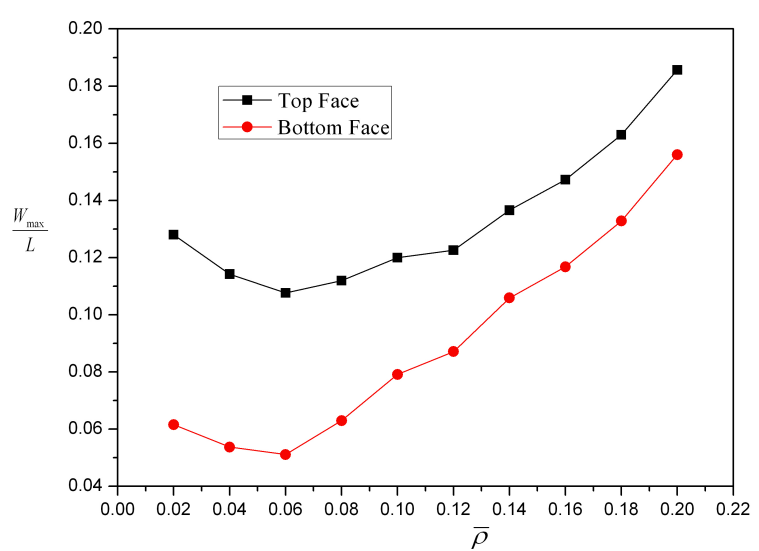

(a)

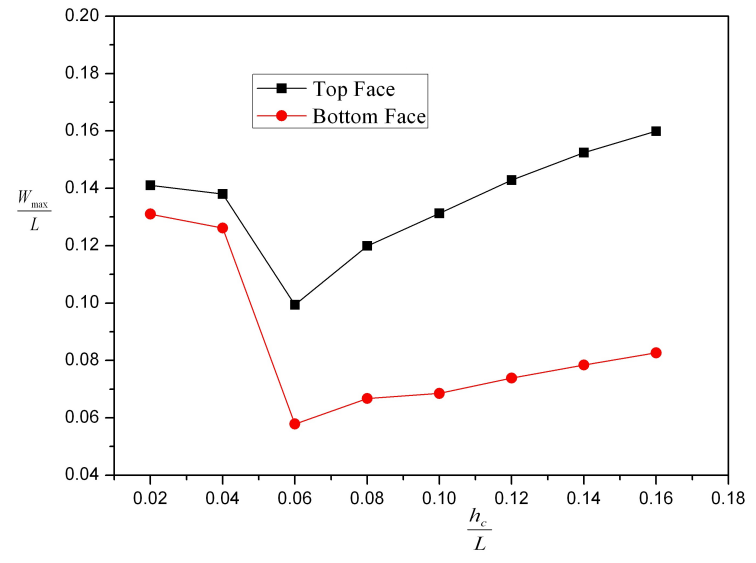

(b)

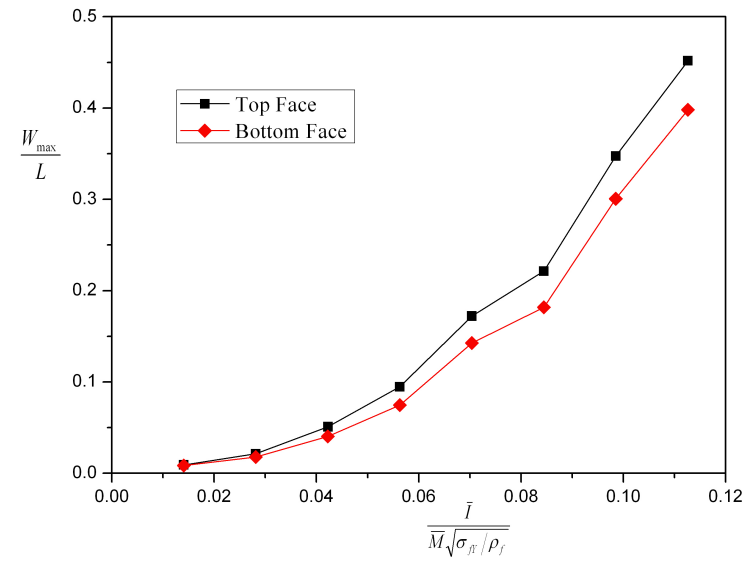

(c)

Figure 7 Normalized maximum deflection $W_{\max } / L$ of the top and bottom face sheet of sandwich shell with $\bar{M} / \rho_{f} L=0.025$ : (a) versus $\bar{\rho}$, (b) versus $h_{c} / L$ and (c) normalized impulse

\section{CONCLUSIONS}

The present study of metallic sandwich shells subjected to air blast loading has exploited the new results of KNR theory, which accounts for nonlinear compressibility of the air and finite shock conditions. It can be found that the compression strain increases approximately linearly with normalized impulse, and reduces with increasing relative density. But it is not sensitive to $h_{f} / h_{c}$ in the studied bound. In addition, numerical results valid that the core compression plays the key role of energy absorption for a sandwich structure subjected to intense air blast. To investigate the coupling effects between the stages of the response of sandwich shell, the velocity-time histories of the center-point of the top and bottom face-sheets of sandwich shells subjected to different impulse levels were presented in the paper. An increase of the impulse delays the equalization of top and bottom face velocities but there is a maximum $t_{e q}=0.068 \mathrm{~ms}$ in the studied bound. An effort to obtain near-optimal configurations for sandwich shells was made to make a compromise between 
strength and weight. It is clear that the optimal structural configuration region of sandwich shells with $\bar{M} / \rho_{f} L=0.025$ subjected to a given normalized impulse $\bar{I} /\left(\bar{M} \sqrt{\sigma_{f Y} / \rho_{f}}\right) \approx 0.07$ is $\bar{\rho}=0.06$ and $h_{c} / L=0.06$.

Acknowledgements This work is supported by the National Natural Science Foundation of China (Grant No. 10972153, 10802055), Program for the Homecomings Foundation and the Top Young Academic Leaders of Higher Learning Institutions of Shanxi. The financial contribution is gratefully acknowledged.

\section{References}

[1] Deshpande VS, Fleck NA. High strain rate compressive behavior of aluminum alloy foams. International Journal of Impact Engineering, 2000, 24: 277-298.

[2] Evans AG, Hutchinson JW, Ashby MF. Multifunctionality of cellular metal systems. Progress in Materials Science, 1999, 43: 171-221.

[3] John Banhart. Metallic foams: challenges and opportunities. Eurofoam 2000, Editors: P. Zitha, J. Banhart, G. Verbist (MIT-Verlag Bremen, 2000), pp. 13-20.

[4] Fleck NA, Deshpande VS. The resistance of clamped sandwich beams to shock loading. Journal of Applied Mechanics, 2004, 71: 386-401.

[5] Jing L, Wang ZH, Ning JG, Zhao LM. The dynamic response of sandwich beams with open-cell metal foam cores. Composites: Part B, 2011, 42:1-10.

[6] Qiu X, Deshpande VS, Fleck NA. Impulsive loading of clamped monolithic and sandwich beams over a central patch. Journal of the Mechanics and Physics of solids, 2005, 53: 1015-1046.

[7] Jing L, Wang ZH, Ning JG, Zhao LM. The mechanical response of metallic sandwich beams under foam projectile impact loading. Latin American Journal of Solids and Structures, 2011, 8: 107-120.

[8] Vaziri A, Hutchinson JW. Metal sandwich plates subject to intense air shocks. International Journal of Solids and Structures, 2007, 44: 2021-2035.

[9] Zhu F, Zhao LM, Lu GX, Wang ZH. Deformation and failure of blast-loaded metallic sandwich panelsExperimental investigations. International Journal of Impact Engineering, 2008, 35: 937-951.

[10] McShane GJ, Radford DD, Deshpande VS, Fleck NA. The response of clamped sandwich plates with lattice cores subjected to shock loading. European Journal of Mechanics A/Solids, 2006, 25: 215-229.

[11] Shen JH, Lu GX, Wang ZH, Zhao LM. Experiments on curved sandwich panels under blast loading. International Journal of Impact Engineering, 2010, 37: 960-970.

[12] Liu XR, Tian XG, Lu TJ, Liang B. Blast resistance of sandwich-walled hollow cylinders with graded metallic foam cores. Composite Structures, 2012, 94: 2485-2493.

[13] Fatt MSH, Surabhi H. Blast Resistance and Energy Absorption of Foam-Core Cylindrical Sandwich Shells under External Blast. Composite Structures, 2012, doi: http://dx.doi.org/10.1016/j.compstruct.2012.05.013

[14] Frostig Yeoshua, Thomsen OT. Non-linear thermo-mechanical behavior of delaminated curved sandwich panels with a compliant core. International Journal of Solids and Structures, 2011, 48: 2218-2237.

[15] Taylor GI. The pressure and impulse of submarine explosion waves on plates. In: Batchelor GK, editor. The scientific papers of Sir Geoffrey Ingram Taylor. Vol. III: aerodynamics and the mechanics of projectiles and explosions. Cambridge: Cambridge University Press; 1963. p. 287-303. 
[16] Kambouchev N, Noels L, Radovitzky R. Nonlinear compressibility effects in fluid-structure interaction and their implications on the air-blast loading of structures. Journal of Applied Physics100, 2006, 063519:1-11.

[17] Jing L, Wang ZH, Zhao LM. Dynamic response of cylindrical sandwich shells with metallic foam cores under blast loading-Numerical simulations. Composite Structures, 2013, 99: 213-223.

[18] Jing L, Xi CQ, Wang ZH, Zhao LM. Simulation of the dynamic response of metallic cylindrical sandwich shells subjected to projectile impact, submitted for publication.

[19] Jing L, Wang ZH, Zhao LM, Shim VPW. Blast resistance of clamped cylindrical sandwich shells with metallic foam cores. Key Engineering Materials, 2013, 535-536: 461-464.

[20] Jing L, Wang ZH, Zhao LM. Response of metallic cylindrical sandwich shells subjected to projectile impactExperimental investigations, submitted for publication.

[21] Li QM, Magkiriadies I, Harrigan JJ. Compressive strain at the onset of densification of cellular solids. Journal of Cellular Plastics, 2006, 42: 371-392.

[22] Tilbrook MT, Deshpande VS, Fleck NA. The impulsive response of sandwich beams: Analytical and numerical investigation of regimes of behavior. Journal of the Mechanics and Physics of Solids, 2006, 54: 2242-2280.

[23] Liang YM, Spuskanyuk AV, Flores SE, Hayhurst DR, Hutchinson JW. The response of metallic sandwich panels of water blast. Journal of Applied Mechanics, 2007, 74: 81-99.

[24] Xue ZY, Hutchinson JW. A comparative study of impulse-resistant metal sandwich plates. International Journal of Impact Engineering, 2004, 30: 1283-1305. 